\title{
Estratégias de análise do desenvolvimento motor em crianças com dificuldade de aprendizagem: uma revisão integrativa
}

\author{
Strategies for analyzing motor development in children with learning difficulties: an integrative \\ review
}

Estratégias de análisis del desarrollo motor en niños con dificultades de aprendizaje: una revisión integradora

\author{
Maria Patrícia Farias de Carvalho \\ ORCID: https://orcid.org/0000-0002-6391-7006 \\ Faculdade de Ensino Superior do Piauí, Brasil \\ E-mail: patrícia_carvalho2008@hotmail.com \\ Flavia Tasmim Techera Antunes \\ ORCID: https://orcid.org/0000-0003-0620-2984 \\ Universidade Luterana do Brasil, Brasil \\ E-mail: tas.min@ rede.ulbra.br \\ Diane Nogueira Paranhos Amorim \\ ORCID: https://orcid.org/0000-0002-5362-5747 \\ Faculdade de Ensino Superior do Piauí, Brasil \\ E-mail: diannenogueira@hotmail.com \\ Lélia Lilianna Borges de Sousa Macedo \\ ORCID: https://orcid.org/0000-0003-1108-6324 \\ Faculdade de Ensino Superior do Piauí, Brasil \\ E-mail: leliafisio@hotmail.com
}

\begin{abstract}
Resumo
Há uma estreita relação entre o que as crianças são capazes de aprender (cognitivo) com o que sãp capazes de realizar (motor). Intervenções psicomotoras voltadas para desenvolvimento de habilidades motoras específicas pode influenciar positivamente nas dificuldades de aprendizagem. Assim, o objetivo desta revisão foi identificar estratégias de avaliação do desempenho motor em crianças com dificuldades de aprendizagem e sua eficácia. Para isto, uma revisão integrativa foi realizada nas bases de dados PubMed, Lilacs e Scielo, através das palavras-chave. Do total de 244 registros, 5 artigos contemplaram os critérios de inclusão nesta revisão sendo que excluíram-se trabalhos onde a dificuldade de aprendizagem está relacionada a desordem do neurodesenvolvimento ou distúrbios neurológicos. As estratégias de avaliação e encontradas foram: tarefas de motricidade fina, global, equilíbrio, organização espacial, temporal, lateralidade e esquema corporal, avaliação da escrita, leitura e aritmética. Conclusão: a avaliação das habilidades motoras é bem descrita como estratégia preventiva e efetiva para minorar as dificuldades de aprendizagem, em crianças com deficiência de aprendizagem.
\end{abstract}

Palavras-chave: Desempenho motor; Crianças; Dificuldade de aprendizagem.

\begin{abstract}
There is a close relationship between what the children are able to learn (cognitive) and what they are able to achieve (motor). Psychomotor interventions aim the development of specific motor skills, and it can positively influence learning difficulties. Thus, the aim of this review was to identify strategies to assess motor performance in children with learning difficulties. For this, an integrative review was carried out in the PubMed, Lilacs and Scielo databases, using the keywords. From a total of 244 records, 5 articles met the inclusion criteria in this review. Several studies were excluded because the learning disability is related to neurodevelopmental disorder or neurological disorders. The assessment strategies found were: fine motor, global, balance, spatial and temporal organization, laterality and body scheme tasks, writing assessment, reading and arithmetic. Conclusion: the assessment of motor skills is well described as a preventive and effective strategy to alleviate learning difficulties in children with learning disabilities.
\end{abstract}

Keywords: Motor performance; Kids; Learning difficulty.

\section{Resumen}

Existe una estrecha relación entre lo que el niño es capaz de aprender (cognitivo) y lo que es capaz de lograr (motor). Las intervenciones psicomotoras destinadas a desarrollar habilidades motoras específicas pueden influir positivamente en las dificultades de aprendizaje. Por lo tanto, el objetivo de esta revisión fue identificar estrategias para evaluar el 
desempeño motor en niños con dificultades de aprendizaje. Para ello, se realizó una revisión integradora en las bases de datos PubMed, Lilacs y Scielo, utilizando las palabras clave. De un total de 244 registros, 5 artículos cumplieron los criterios de inclusión en esta revisión y se excluyeron los estudios donde la discapacidad de aprendizaje está relacionada con un trastorno del desarrollo neurológico o trastornos neurológicos. Las estrategias de evaluación y las encontradas fueron: motricidad fina, global, equilibrio, organización espacial y temporal, tareas de lateralidad y esquema corporal, evaluación de escritura, lectura y aritmética. Conclusión: la analisis de las habilidades motoras está bien descrita como una estrategia preventiva y efectiva para aliviar las dificultades de aprendizaje en niños con discapacidades de aprendizaje.

Palabras clave: Rendimiento motor; Niños; Dificultad de aprendizaje.

\section{Introdução}

É na infância que ocorre um incremento de habilidades motoras, que possibilitam à criança um amplo domínio do seu corpo em diferentes atividades (Santos et al., 2004). O desenvolvimento humano é decorrente da interação de vários fatores, nos quais estão implicados aspectos psicossociais, cognitivos, motores e afetivos (Rosa Neto et al., 2010a). Assim, há uma estreita relação entre o que a criança é capaz de aprender (cognitivo) com o que é capaz de realizar (motor). Isso significa que, ao conquistar um bom controle motor, a criança construirá as noções básicas para o seu desenvolvimento intelectual (Rosa Neto et al., 2010b). Portanto, a intervenção psicomotora voltada para desenvolvimento de habilidades motoras específicas pode influenciar positivamente nas dificuldades de aprendizagem (Nunes et al., 2014).

Em estudo realizado por Medina e Marques (2010), crianças com dificuldade de aprendizagem apresentaram desenvolvimento motor abaixo do ideal para idade, sustentando a ideia de que o déficit motor pode ocasionar uma dificuldade de aprendizagem. Neste sentido, a identificação precoce dos déficits motores pode fornecer ao indivíduo acometido uma maior chance de êxito e, consequentemente, uma melhor qualidade de vida por possibilitar o desenvolvimento de programas de intervenção para esta população, tanto no campo da saúde como da educação (Poeta \& Rosa Neto, 2005; Vieira et al., 2009).

No contexto escolar, crianças com dificuldades motoras são excluídas por seus colegas em brincadeiras e jogos, por não apresentarem competências suficientes nos movimentos motores (Santos \& Vieira, 2013). O desenvolvimento motor na infância caracteriza-se pela aquisição de um amplo espectro de habilidades motoras, que possibilita a criança um melhor domínio do seu corpo em diferentes posturas (estáticas e dinâmicas), locomover-se pelo meio ambiente de formas variadas (andar, correr, saltar, etc.) e manipular objetos e instrumentos diversos (receber uma bola, arremessar uma pedra, chutar, escrever, etc.) (Santos et al., 2004).

Além do domínio motor e cognitivo, crianças com transtorno do desenvolvimento da coordenação também podem apresentar problemas emocionais como baixa autoestima, ansiedade e depressão que podem levá-las a um isolamento social (Wilson 2020; Rivard et al., 2007; Zwicker et al., 2009; Zwicker et al., 2012). Frente a isso, o presente estudo teve como objetivo revisar estratégias de avaliação de habilidades motoras no enfrentamento de dificuldades de aprendizagem e sua eficácia em minorá-las.

\section{Metodologia}

Trata-se de revisão integrativa para avaliar e sintetizar os resultados de pesquisas sobre temática específica (Mendes et al., 2008). Para elaboração da questão de pesquisa da utilizou-se a estratégia PICO (acrônimo para P: população/pacientes; I: intervenção; C: comparação/controle; O: desfecho/outcome). Assim, a questão da pesquisa delimitada foi : "Como avaliar as habilidades motoras em crianças com dificuldades de aprendizagem?" Nela, o primeiro elemento da estratégia (P) consiste em crianças; o segundo (I), o desempenho motor; e o terceiro elemento (O) dificuldade de aprendizagem. Nesta revisão integrativa, o terceiro elemento, ou seja, a comparação, não foi utilizada.

A busca dos estudos ocorreu de agosto a setembro de 2021, nas seguintes bases de dados: National Library of 
Medicine National Institutes of Health (NLM NIH - Pubmed), Literatura Latino-Americana e do Caribe em Ciências da Saúde (LILACS) e Scientific Electronic Library Online (Scielo). Os descritores controlados selecionados nos Descritores em Ciências da Saúde (DECS) da Biblioteca Virtual em Saúde (BVS) e MeSH Databse foram children, psychomotor performance, e learning disability, os quais foram combinados de diferentes formas para garantir busca ampla através dos cruzamentos em todas as bases de dados.

Os critérios de inclusão estabelecidos para os estudos foram artigos que abordavam o desempenho motor em crianças com dificuldade de aprendizagem. As revisões tradicionais de literatura, revisão sistemática, carta-reposta e editoriais foram excluídos da amostra, assim como artigos que tratavam de desordem do neurodesenvolvimento e desordens neurológicos. Houve também limitação do idioma em português e inglês devido o extensa produção científica sobre o desempenho motor.

Na primeira análise, após a leitura de títulos e resumos dos estudos primários ( $\mathrm{n}=244)$, os estudos que não indicavam nenhum desempenho motor relacionado a dificuldade de aprendizagem foram excluídos (n=229) (Figura 1). Dentre eles: artigos secundários sobre dificuldade de aprendizagem relacionada a desordem do neurodesenvolvimento ou distúrbios neurológicos que não atendiam à pergunta da pesquisa, e temas não relacionados. Na segunda análise, não foram encontrados artigos duplicados. As análises foram desenvolvidas de forma independente por duas revisoras.

Para extração dos dados, utilizou-se instrumentos elaborados pelas pesquisadoras, o qual foi composto por itens relativos a identificação dos artigos, características metodológicas e avaliação em rigor metodológico. A análise dos resultados foi realizada de forma descritiva, sendo apresentada a síntese de cada estudo incluído na revisão e comparações entre as pesquisas incluídas destacando as semelhanças e diferença na Tabela 1.

Figura 1. Diagramação dos resultados da seleção de artigos incluídos nesta revisão integrativa.

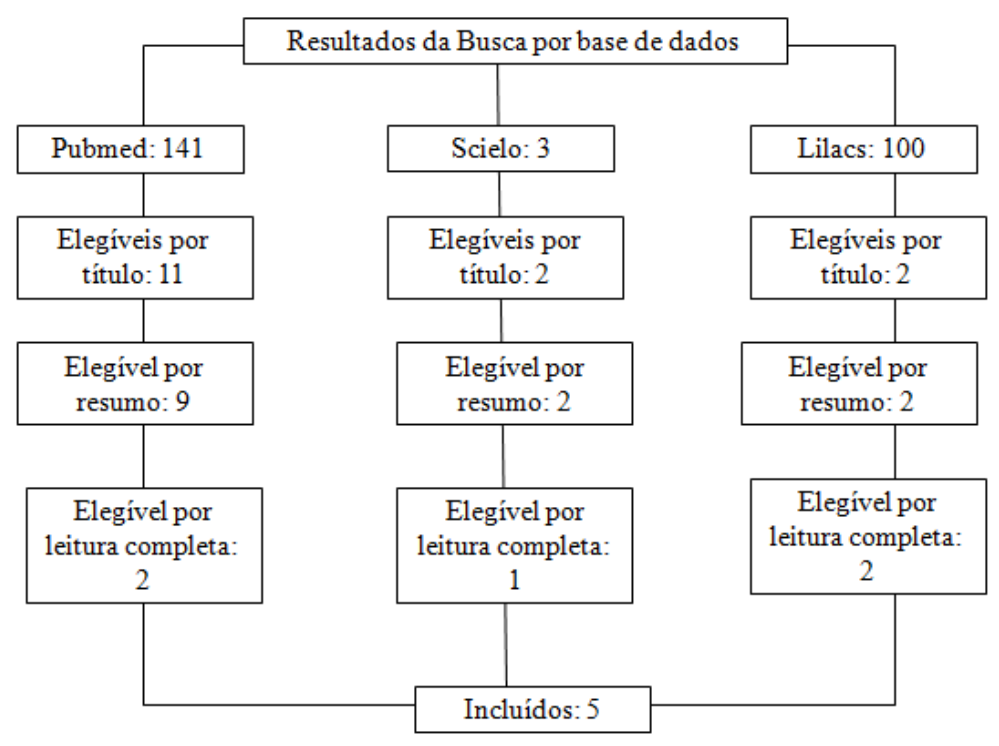

Fonte: Autores.

\section{Resultados}

A amostra da revisão integrativa foi composta de 5 estudos exploratórios, sendo estes publicados majoritariamente em 2017 e 2016. Em relação ao idioma, 3 foram publicados em inglês e dois em português. Na Tabela 1 são demonstradas as principais informações extraídas dos estudos primários incluídos na revisão. 
Tabela 1. Síntese dos estudos incluídos na revisão integrativa.

\begin{tabular}{|c|c|c|c|}
\hline Autor/Ano & Objetivo & Estratégia & Principais resultados \\
\hline Cardillo et al., (2017) & $\begin{array}{l}\text { Investigar o efeito da organização perceptual } \\
\text { e dificuldades de tarefas, comparando } \\
\text { crianças com sintomas de deficiência de } \\
\text { aprendizagem não verbal ou dislexia com } \\
\text { crianças controle. }\end{array}$ & $\begin{array}{l}\text { A tarefa baseada em computador foi administrada usando } \\
\text { um laptop, e o procedimento experimental foi } \\
\text { programado com o software E-Prime. }\end{array}$ & $\begin{array}{l}\text { Importância de examinar os processos visuoespaciais em crianças com } \\
\text { deficiência de aprendizagem, e a utilidade das diferentes versões da } \\
\text { tarefa de design de bloco em distinguir entre as modalidades de } \\
\text { processamento global e local. }\end{array}$ \\
\hline $\begin{array}{l}\text { Niechwiej-Szwedo et } \\
\text { al., (2017) }\end{array}$ & $\begin{array}{l}\text { Avaliar o desempenho das habilidades } \\
\text { motoras em crianças com dificuldades de } \\
\text { leitura e associar a visão binocular. }\end{array}$ & $\begin{array}{l}\text { A avaliação das habilidades motoras envolveu duas } \\
\text { tarefas de manipulação: rosqueamento do cordão e tarefa } \\
\text { com pranchas de pinos }\end{array}$ & $\begin{array}{l}\text { Crianças com dificuldades na leitura tiveram pior desempenho apenas } \\
\text { na tarefa do rosqueamento do cordão em comparação com o grupo } \\
\text { controle. } \\
\text { O desempenho das tarefas motoras foi associado a facilidade de } \\
\text { acomodação reduzida (monocular e binocular) o que mostra uma } \\
\text { associação entre desempenho das habilidades motoras reduzida e as } \\
\text { dificuldades de leitura. }\end{array}$ \\
\hline da Silva et al., (2012) & $\begin{array}{l}\text { Verificar as dificuldades motoras e de } \\
\text { aprendizagem em escolares com baixo } \\
\text { desempenho escolar. }\end{array}$ & $\begin{array}{l}\text { Foi realizado o teste de desempenho escolar (TDE) } \\
\text { através de três tipos de avaliações: escrita; aritmética e } \\
\text { leitura e o teste de bateria de avaliação motora para } \\
\text { criança. }\end{array}$ & $\begin{array}{l}\text { Com exceção do teste aritmética os participantes obtiveram um } \\
\text { desempenho escolar abaixo da média para ambos os sexos. } \\
\text { Não foram encontradas diferenças estatisticamente significantes ao } \\
\text { comparar os indicativos de dificuldades de aprendizagem. }\end{array}$ \\
\hline $\begin{array}{l}\text { Rosa e Neto et al, } \\
(2011)\end{array}$ & $\begin{array}{l}\text { Verificar o desenvolvimento do esquema } \\
\text { corporal de escolares na faixa etária de } 6 \text { a } 10 \\
\text { anos com queixa de dificuldades de } \\
\text { aprendizagem }\end{array}$ & Foi utilizado a escala de desenvolvimento motor -EDM & $\begin{array}{l}\text { Evidencia que a grande maioria da amostra teve o desenvolvimento } \\
\text { motor classificado como muito } \\
\text { inferior e inferior, e nenhuma criança atingiu níveis além do normal } \\
\text { baixo. } \\
\text { Na sua maioria, o esquema corporal esteve entre a principal ou as } \\
\text { principais áreas que apresentaram maior déficit motor em } \\
\text { relação ao esperado para a idade da criança. }\end{array}$ \\
\hline Soares et al., (2015) & $\begin{array}{l}\text { Analisar o desempenho motor de crianças } \\
\text { com queixas de dificuldades de aprendizagem } \\
\text { após a intervenção de um programa de } \\
\text { atividade motora. }\end{array}$ & $\begin{array}{l}\text { A avaliação motora, foi realizada por meio da EDM, } \\
\text { função motora global, equilíbrio, esquema corporal, } \\
\text { organização espacial, organização temporal e } \\
\text { lateralidade. }\end{array}$ & $\begin{array}{l}\text { Somente crianças com queixas de dificuldades de aprendizagem que } \\
\text { foram submetidas à intervenção obtiveram progressão } \\
\text { percentual superior a } 20 \% \text {. Crianças com e sem dificuldades que } \\
\text { participaram da intervenção apresentaram progressão entre } 10 \text { e } 20 \% \text {. }\end{array}$ \\
\hline
\end{tabular}

Fonte: Autores (2021). 


\section{Discussão}

Há evidências de estratégias psicomotoras para reduzir as dificuldades motoras em indivíduos com dificuldades de aprendizagem e minorar os déficits intelectuais, as quais são principalmente intervenções que estimulam o desempenho motor. O presente estudo reuniu dados sobre a eficácia destas avaliações que utilizaram o teste de habilidades motoras de diferentes formas, comparando-as com as dificuldades de aprendizagem através de testes de desempenho escolar. Foi possível observar que os resultados das habilidades motoras foram significativos não apenas para crianças com dificuldade de aprendizado, mas também para crianças sem dificuldade de aprendizagem (Soares et al., 2015) comparando as habilidades motoras e a integração oculomotora.

Os componentes da aprendizagem motora exercem influências significativas na aquisição de habilidades de aprendizagem cognitiva, pois estas capacidades são solicitadas posteriormente no processo de aprendizagem da leitura e escrita (Freitas, 2008; Medina et al, 2006; Medina-papst, Marques, 2010). Assim, as habilidades motoras podem ser consideradas integradas, tendo relação com as funções cognitivas, executivas e sensoriais (Delgado et al., 2016, Wilson et al., 2020). Essa associação do desempenho motor com dificuldade de aprendizado reflete nas relações sociais, emocionais e acadêmicas, mas por outro lado, o movimento e o brincar espontaneamente precedem as atividades mais estruturadas de aprendizagem (Rosa Neto et al., 2011). A importância dessa intervenção motora refere-se à relação sujeito e o meio, que é uma forma equilibrada e fundamental para o desenvolvimento da criança (Rosa Neto et al., 2006).

Kashfi et al. (2019) analisaram o efeito de uma intervenção motora baseada na abordagem de aprendizagem de atenção, equilíbrio e coordenação sobre proficiência motora e funções executivas em crianças com deficiência de aprendizagem. Estudantes do ensino fundamental do sexo masculino de 7 a 9 anos foram recrutados e foi observado que a intervenção motora três vezes por semana durante oito semanas mostrou melhorara significativamente suas habilidades motoras e a maioria das medidas das funções executivas das crianças. Por outro lado, Bayazit et al. (2021), conduziu estudo para determinar se algumas habilidades psicomotoras de crianças com deficiência de aprendizagem específica se desenvolvem com a educação do movimento. Aqui, um programa de treinamento de movimentos (movimentos locomotores, manipulativos e de equilíbrio, que incluem equilíbrio estático infantil, equilíbrio dinâmico, raquete para bola, arremesso para o alvo, salto cruzado, drible, troca de bola e ultrapassagem de obstáculos) 60 minutos por dia, 3 vezes na semana durante 8 semanas mostrou efeitos positivos em algumas habilidades psicomotoras em crianças de 7 a 14 anos com dificuldades de aprendizagem específicas.

Habilidades motoras visuais, que são habilidades cognitivas complexas, são difíceis para crianças com deficiência de aprendizagem não-verbal (Cardillo et al., 2017), refletindo problemas de planejamento, organização e raciocínio visuoespacial, bem como habilidades motoras visuais (Semrud-Clikeman \& Glass, 2010). As estratégias para o desempenho motor que envolve a motricidade fina, global, equilíbrio, esquema corporal, organização espacial, temporal e lateralidade demonstraramse práticos para serem utilizadas em crianças em idade escolar. Embora não tenham sido identificadas pesquisas de aplicação de tais estratégias com maior número de atividades motoras, sugere-se que o desenvolvimento de estudos a respeito proporcionaria a possibilidade de diminuir as limitações relacionadas às tarefas que envolvem as habilidades motoras visuais (Cardillo et al., 2017). Os autores ainda destacam que a organização visuo-espacial pode ser potencialmente melhorada através do processamento cognitivo local e global, possibilitando identificar as dificuldades nas tarefas que envolvam o aspecto visuoespacial.

Ainda com relação a ação de estratégias sobre a organização visuo-espacial, crianças com baixa habilidade perceptiva ou motora pode refletir dificuldades de aprendizado da leitura, escrita e matemática (Berry \& O’Conor, 2010). Estes achados são identificados também no estudo de Ibrahim et al, (2019), que avaliaram escolares com dificuldades de aprendizagem específicas, e observaram dificuldades de movimento principalmente na destreza manual em comparação as outras crianças. A 
acomodação da visão está diretamente relacionada com a convergência, já que existe uma sincinesia entre ambas (Sá \& Plutt, 2001). Isto impactaria diretamente nas dificuldades de leitura e consequentemente sobre o desenvolvimento de intervenções adequadas e na qualidade de vida de crianças com dificuldades de leitura e reduzida habilidade motora (Niechwiej-Szwedo et al., 2017).

A EDM proposta por Rosa Neto (2002) envolve testes de motricidade fina e global, equilíbrio, lateralidade, organização espacial, temporal e esquema corporal. É um instrumento de avaliação eficaz, bem como utilizado com maior frequência em um ambiente escolar, a fim de prevenir um avanço maior em casos em que o problema motor já existe e também evitar um possível atraso inicial (Santos et al., 2021). Nessa escala, as habilidades cognitivas são adquiridas através de ações desenvolvidas pelo corpo. As estratégias da utilização da escala podem ser eficazes e utilizadas como ferramenta clínica para auxiliar no acompanhamento do desenvolvimento motor e cognitivo. Isto pode, consequentemente, resultar em diminuição dos déficits de aprendizagem, reduzindo o baixo desempenho acadêmico e risco de evasão escolar. Os autores não discutem, no entanto, os testes mais eficazes para avaliação da dificuldade de aprendizagem. A avaliação do desenvolvimento motor por estimular o controle motor, pode ser uma alternativa para construir as noções básicas do desenvolvimento intelectual, uma vez que o movimento é essencial para o desenvolvimento das crianças, permitindo as interações em ambientes sociais e físicos e favorece a aquisição de diferentes tipos de experiências (Deconinck et al., 2006). Ainda sobre a EDM, Santos et al., (2019) revela que a EDM está sendo utilizada no Brasil, em diversos contextos, apresentando resultados claros e estatisticamente consistentes, embora as metodologias dos estudos não atendam plenamente padrões de qualidade metodológica. Além disso, a EDM foi validada como sendo uma maneira concisa, parcimoniosa e confiável para avaliar as habilidades de desenvolvimento motor em crianças de 4, 5 e 6 anos (Okuda et al., 2020).

O único estudo encontrado em que se verificou o esquema corporal, com a finalidade de obter experiências corporais através do movimento do corpo, empregou a EDM (Rosa Neto et al., 2011). Essa escala foi adotada com crianças na faixa etária de 6 a 10 anos, com diferentes desempenhos escolares, assim foram realizados três testes motores. Observou-se que estratégias que utilizam o esquema corporal configuram-se como uma forma de equilíbrio entre o sujeito e o meio. Os pesquisadores incentivaram a realização da avaliação do desenvolvimento motor de forma precoce, para desenvolvimento harmonioso dos aspectos motores, físicos e cognitivos. No entanto, não foram encontradas estratégias que utilizassem apenas o esquema corporal para o auxílio das dificuldades de aprendizagem, mesmo sabendo da conexão dessa habilidade com a aquisição de habilidades cognitivas, pois estas capacidades são solicitadas posteriormente no processo de aprendizagem da leitura e escrita (Freitas, 2008; Medina et al., 2006; Medina-papst \& Marques, 2010; Rosa Neto et al., 2010b).

Um dos grandes elos no desenvolvimento infantil de acordo com Piek et al., (2008) é o desenvolvimento motor e cognitivo, juntamente com o desenvolvimento socioemocional, os quais formam as três áreas de desenvolvimento interrelacionadas nos primeiros anos de aprendizagem e desenvolvimento. Á vista disso, estratégias como o desenvolvimento precoce das habilidades motoras e cognitiva mostram-se seguros e eficazes, melhorando a média do rendimento escolar aos 16 anos (Taanila et al., 2005) e o desempenho intelectual aos 8, 26 e 53 anos (Murray et al., 2007).

Na presente revisão observa-se a escassez de pesquisas com a padronização de estratégias para avaliar a dificuldade de aprendizagem em crianças com dificuldade motora, embora corroborem com descobertas recentes na área da psicomotricidade. Frente a isso, há necessidade de investigação futura sobre a problematização. Houve a inclusão de um estudo que avaliou somente o esquema corporal no conjunto de testes de avaliação motora, o qual se justifica pelo uso criterioso de metodologia da busca e devido a relevância de seus resultados para a elucidação da pergunta da pesquisa.

\section{Considerações Finais}

A avaliação de estratégias para reduzir as dificuldades de aprendizagem, consistiu, sobretudo, na comparação com os 
principais testes motores usuais de rotina. As habilidades motoras atuam em amplo domínio do corpo e em diferentes posturas estáticas e dinâmicas, que vâo ativar áreas do cérebro responsáveis pelas habilidades cognitivas. Estas focam no esquema corporal e atuam na relação da capacidade de aprender (cognitivo) com o que é capaz de realizar (motor). Portanto, a avaliação das habilidades motoras com as dificuldades de aprendizagem, por sua vez, mostrou-se uma estratégia eficaz e segura para reduzir deficiência de aprendizagem bem como aumentar o desempenho motor, devendo ser realizada por profissionais da saúde e baseados em avaliação criteriosa e individualizada de cada criança. Mesmo assim, há necessidade de intensificar esforços para o desenvolvimento de pesquisa com metodologias capazes de produzir evidências fortes relativas a este tema, sobretudo na prática com crianças com dificuldades de aprendizagem e que apresentam atrasos motores. Em suma, o uso de instrumentos para avaliar a psicomotricidade na educação infantil e fundamental carece de estudos de validação. Ademais, os instrumentos existentes devem ser analisados pela qualidade psicométrica, especialmente em um contexto educacional. Estudos longitudinais mais longos também são necessários para discriminar os benefícios dos métodos de avaliação.

\section{Referências}

Bayazit, B., Tezel, F., \& Çakmak, G. (2021). The Effect of Movement Education Studies Applied to Children with Specific Learning Disability on Psychomotor Development. Pamukkale Journal if Sport Sciences, 12(1), 29-40.

Berry, D., \& O'Connor, E. (2010). Behavioral risk, teacher-child relationships, and social skill development across middle childhood: A child-byenvironment analysis of change. Journal of Applied Developmental Psychology, 31(1),1-14. 10.1016/j.appdev.2009.05.001

Cardillo, R., Mammarella, I. C., Garcia, R. B., \& Cornoldi, C. (2017). Local and global processing in block design tasks in children with dyslexia or nonverbal learning disability. Research in developmental disabilities, 64, 96-107. https://doi.org/10.1016/j.ridd.2017.03.011

da Silva, J., Beltrame, T. S., de Oliveira, A. V. P., \& Sperandio, F. F. (2012). Dificuldades motoras e de aprendizagem em crianças com baixo desempenho escolar. Journal of Human Growth and Development, 22 (1), 41-46. https://doi.org/10.7322/jhgd.20048

Deconinck, F. J., De Clercq, D., Savelsbergh, G. J., Van Coster, R., Oostra, A., Dewitte, G., \& Lenoir, M. (2006). Differences in gait between children with and without developmental coordination disorder. Motor control, 10(2), 125-142. https://doi.org/10.1123/mcj.10.2.125

Delgado, L., Montes, R., \& Prieto, J. A. (2016) Prevalência de retardo psicomotor e sua relação com o perfil sensorial em pré-escolares. Journal of Human Growth and Development, 26 (3), 323-330.

Freitas, N. K. (2008). Esquema corporal, imagem visual e representação do próprio corpo: questões teórico-conceituais. Ciências e Cognição, 13(3), 318-324.

Ibrahim, S., Harun, D., Kadar, M., Rasdi, H. F. M., Bharudin, N. S., \& Hui, E. J. T. (2019). Motor performance and functional mobility in children with specific learning disabilities. The Medical Journal of Malaysia, 74 (1), 34-39.

Kashfi, T. E., Sohrabi, M., Kakhki, A. S., Mashhadi, A., \& Nooghabi, M. J. (2019). Effects of a Motor Intervention Program on Motor Skills and Executive Functions in Children With Learning Disabilities. Perceptual and Motor Skills, 126(2):003151251983681

Medina, J., Rosa, G. K. B., \& Marques, I. (2006). Desenvolvimento da organização temporal de crianças com dificuldades de aprendizagem. Revista da Educação Física/ UEM, 17(1), 107-116.

Medina-Papst, J., \& Marques, I. (2010) Avaliação do desenvolvimento motor de crianças com dificuldades de aprendizagem. Revista Brasileira de Cineantropometria \& Desempenho Humano, 12 (1), 36-42.

Mendes, K. S., Silveira, R. C. C. P., \& Galvão, C. M. (2008). Revisão integrativa: método de pesquisa para a incorporação de evidências na saúde e na enfermagem. Texto \& contexto-enfermagem, 17, 758-764.

Murray, E. A., O'Doherty, J. P., \& Schoenbaum, G. (2007). What we know and do not know about the functions of the orbitofrontal cortex after 20 years of cross-species studies. The Journal of neuroscience: the official journal of the Society for Neuroscience, 27(31), 8166-8169. https://doi.org/10.1523/JNEUROSCI.1556-07.2007

Niechwiej-Szwedo, E., Alramis, F., \& Christian, L. W. (2017). Association between fine motor skills and binocular visual function in children with reading difficulties. Human movement science, 56(Pt B), 1-10.

Nunes, L. C., Neves, D., de Freitas, T. D., Floriano, P. M., \& Simone, L. (2014). Perfil de estudantes dos anos iniciais com baixo rendimento escolar: importância da educação física na escola. Revista Brasileira de Ciência e Movimento, 22 (2), 36-46.

Okuda, P. M. M., Felix, E., Cogo-Moreira, H., Liu, T., Surkan, P. J., Martins, S. S., et al. (2020). Construct validity of the Motor Development Scale (MDS). Trends in Psychiatry and Psychotherapy, 42(1), 82-85.

Piek, J. P., Dawson, L., Smith, L. M., \& Gasson, N. (2008). The role of early fine and gross motor development on later motor and cognitive ability. Human movement science, 27(5), 668-681. 
Poeta, L. S., \& Rosa Neto, F. (2005). Intervenção motora em uma criança com transtorno do déficit de atenção/hiperatividade (TDAH). Lecturas Educación Física y Deportes, 10 (89).

Rivard, L. M., Missiuna, C., Hanna, S., \& Wishart, L. (2007). Understanding teachers' perceptions of the motor difficulties of children with developmental coordination disorder (DCD). The British journal of educational psychology, 77(Pt 3), 633-648.

Rosa Neto, F. (2002). Escala de Desenvolvimento Motor (EDM): manual de avaliação motora. Artmed.

Rosa Neto, F., Amaro, K. N, Prestes, D. B. \& Arab. C. (2011). O esquema corporal de crianças com dificuldade de aprendizagem. Psicologia Escolar e Educacional, 15, 15-22.

Rosa Neto, F., Caon, G., Bissani, C, Silva, C. A., Souza, M., \& Silva, L. (2006). Características neuropsicomotoras de crianças de alto risco neurológico atentidas em um programa de fallowup. Pediatria Moderna, 42 (2). 79-85.

Rosa Neto, F., dos Santos A. P. M., Weiss, S. L., \& Amaro, K. N. (2010). Análise da consistência interna dos testes de motricidade fina da EDM - Escala de Desenvolvimento Motor. R. da Educação Física /UEM, 21(2), 191-197.

Rosa Neto, F., dos Santos A. P. M., Xavier R. F. C., \& Amaro. K. N. (2010). A Importância da avaliação motora em escolares: análise da confiabilidade da Escala de Desenvolvimento Motor. Revista Brasileira de Cineantropometria \& Desempenho Humano, 12, (6) 422-427.

Sá, L. C. F., \& Plutt, M. (2001). Acomodação. Arquivos Brasileiros de Oftalmologia, 64 (5), 481-483.

Santos, I. S., Baretta, E., Baretta, M., Souza, R., Alberti, A., \& Grigollo, L. R. (2021). Avaliação da motricidade fina, global e do equilíbrio em escolares de Água Doce, SC. Brazilian Journal of Development, 7 (1), 4931-4941.

Santos, M. C. S., Shimano, S. G. N., Arapujo, L. G. O., \& Pereira, K (2019). Application od motor development scale: na integrative review. Revista CEFAC, $21(4)$, e9918

Santos, S., Dantas, L., \& Oliveira, J. A. (2004). Desenvolvimento motor de crianças, de idosos, e de pessoas com transtorno da coordenação. Revista Paulista de Educação Física, 18, 33-44.

Santos, V. A. P., \& Vieira, J. L. L. (2013). Prevalência de desordem coordenativa desenvolvimento em crianças com 7 a 10 anos de idade. Revista Brasileira de Cineantropometria \& Desempenho Humano, 15 (2). https://doi.org/10.5007/1980-0037.2013v15n2p233

Semrud-clikeman, M., \& Glass, K. (2010) The relation of humor and child development: Social, adaptive, and emotional aspects. Journal of Child Neurology, 25 (10), 1248-1260.

Soares, D. B., Porto, E., de Marco, A., Azoni, C. A. S., \& Capelatto, I. V. (2015) Influence of the physical activity on motor performance of children with learning difficulties. Revista CEFAC, 17, 1132-1142.

Taanila, A., Murray, G. K., Jokelainen, J., Isohanni, M., \& Rantakallio, P. (2005). Infant developmental milestones: a 31-year follow-up. Developmental medicine and child neurology, 47(9), 581-586.

Vieira, S. S. C. (2009). Avaliação de programas de educação continuada: análise da percepção do aluno e do seu modelo de decisão para escolha de programas de especialização para executivos no Brasil. Tese de Doutorado. Universidade de São Paulo.

Wilson, P., Ruddock, S., Rahimi-Golskhandan, S., Piek, J., Sugden, D., Green, D., \& Steerbergen, B. (2020). Cognitive and motor function in developmental coordination disorder. Developmental Medicine \& Child Neurology, 62 (11), 1317-1323.

Zwicker, J. G., Missiuna, C., \& Boyd, L. A. (2009). Neural correlates of developmental coordination disorder: a review of hypotheses. Journal of child neurology, 24(10), 1273-1281.

Zwicker, J. G., Missiuna, C., Harris, S. R., \& Boyd, L. A. (2012). Developmental coordination disorder: a review and update. European journal of paediatric neurology: EJPN: official journal of the European Paediatric Neurology Society, 16(6), 573-581. 